\title{
Correspondence
}

\section{Epidural fentanyl and C-section}

To the Editor:

Breen and Janzen have recently reported no intraoperative maternal benefit from the use of epidural fentanyl during the initiation of epidural block for Caesarean section. ' This is not consistent with our clinical experience nor that reported by others and this paper deserves close scrutiny on that account. In the power calculation used to determine sample size, the authors assumed that early administration of epidural fentanyl would result in a fourfold reduction in the number of patients requiring supplemental intraoperative analgesia during Caesarean section. This is an unusually large difference in outcome to be used in the power analysis. This assumption will result in a small sample population being studied and a clinically important difference in outcome, albeit not a four-fold difference, may be missed (type 2 error). For example, if early administration of epidural fentanyl results in a reduction by half in the number of patients requiring supplemental analgesia, both a realistic goal and an important clinical finding, the number of patients studied in this protocol would have been inadequate to demonstrate such a difference. The recommended sample size for this latter scenario would be close to double the number of patients actually studied in the Breen/Janzen protocol.

The authors grouped the intraoperative analgesic supplements that were administered according to whether the supplement was given before or after delivery and to the actual supplement given. It makes more sense, clinically and statistically, to generate a summary statistic representing all the supplemental analgesic interventions within each group during the intraoperative period and to compare that statistic between the groups. Arbitrarily subgrouping analgesic requirements in this manner also increases the likelihood of a type 2 error.

Had the authors studied a larger sample population, provided the trends reported in their paper continued, and if a summary statistic was generated for the total intraoperative analgesic supplements given, it is likely that the authors' conclusion would have been quite different. This paper should not discourage clinicians from employing a very useful technique, the administration of a lipidsoluble narcotic during initiation of epidural blockade for Caesarean section to enhance intraoperative anaesthesia.
Edward T. Crosby MD FRCPC

Department of Anaesthesia

Ottawa General Hospital

University of Ottawa

\section{REFERENCE}

1 Breen TW, Janzen JA. Epidural fentanyl and Caesarean section: when should fentanyl be given. Can $\mathbf{J}$ Anaesth 1992; 39: 317-22.

\section{$R E P L Y$}

We appreciate Dr. Crosby's interest in our article. ${ }^{1}$ He makes several valid points, but we do not agree with all of his statements. We reported that the improved quality of epidural anaesthesia during Caesarean section did not differ whether epidural fentanyl was given before or after delivery of the infant. To our knowledge, no other study has addressed whether giving fentanyl while establishing epidural blockade offers advantages compared with giving it after delivery.

Dr. Crosby is correct that an error was made when performing the original power analysis. To detect the hypothesized differences a sample size of approximately 50 patients per group would have been needed. To detect a smaller difference as Dr. Crosby suggests, the sample size would need to be approximately 150 patients per group.

A summary statistic should have been used for the data in Table IV. It is not obvious from the Table how many patients received supplemental analgesics as some patients received $\mathrm{N}_{2} \mathrm{O}$ and ketamine, $\mathrm{N}_{2} \mathrm{O}$ and fentanyl or $\mathrm{N}_{2} \mathrm{O}$, ketamine and fentanyl. There was a trend to less supplemental analgesic use by patients who received epidural fentanyl before delivery. If the trend had continued, approximately 70 patients per group would have been needed for the results to have reached statistical significance at the $P<0.05$ level. Except for this trend, evidence of benefit from fentanyl given before delivery was lacking (we did not see more rapid establishment of a $T_{4}$ level of blockade, less need for lidocaine, less shivering or less nausea/vomiting). Thus, if a benefit is derived by giving epidural fentanyl before delivery compared to giving it after, the benefit is small.

Terrance W. Breen MD FRCPC

James A. Janzen MD FRCPC

Department of Anaesthesia

Foothills Hospital

University of Calgary

REFERENCE

1 Breen TW, Janzen $J A$. Epidural fentanyl and Caesarean section: when should fentanyl be given? Can $J$ Anaesth 1992; 39: 317-22. 